\section{Tratamento do Diabetes Mellitus Tipo 1 com Bomba de Infusão Subcutânea Continua de Insulina e Insulina Lispro}

\section{RESUMO}

Objetivo: Avaliar a eficácia do tratamento do diabetes mellitus tipo 1 por um período de 18 meses, em 17 pacientes, utilizando-se um processo de infusão subcutânea contínua de insulina (ISCI) e de um análogo da insulina (insulina lispro) através de uma bomba infusora. Estudamos os seguintes parâmetros: hemoglobina glicosilada, peso, índice de massa corpórea, relação insulina/peso, quantidade de insulina/dia administrada, relação da insulina administrada na forma de bolus (antes das refeições) com a insulina basal e efeitos adversos desta terapia. Após esse período observamos que os pacientes estavam melhor controlados com niveis de HbAlc significativamente reduzidos $(\mathrm{p}<0,05)$. Apresentavam uma redução significativa da relação insulina/peso $(p<0,05)$ e melhor aproveitamento da insulina relacionado a alimentação dado pelo aumento significativo da relação bolus/basal quando comparado com a relação de insulina regular/NPH antes do tratamento $(p<0,05)$.

(Arq Bras Endocrinol Metab 2001;45/5:487-493)

Unitermos: Diabetes mellitus tipo 1; Infusão subcutânea de insulina; Insulina lispro.

\begin{abstract}
Objective: To evaluate the treatment efficacy of type 1 diabetes mellitus during 18 months with a continuous subcutaneous insulin infusion pump and insulin lispro. The following paramethers were studied: glycosylated hemoglobin, weight, body mass index, amount of insulin administered daily, ratio between daily insulin dose and weight, ratio between insulin administered as bolus and as basal and the colateral effects of this form of therapy. After 18 months the patients were in better diabetic control with a significant lower level of glycosylated hemoglobin $(p<0.05)$. They presented a significant reduction in the ratio between insulin daily dose and weight $(p<0.05)$ and a better utilization of insulin related to meal observed by the significant rise in the ratio between insulin administered as bolus and as basal when compared with the ratio of regular and NPH before the SCII treatment $(\mathrm{p}<$ 0.05). (Arq Bras Endocrinol Metab 2001;45/5:487-493)
\end{abstract}

Keywords: Type 1 diabetes mellitus; Continuous subcutaneous insulin infusion; Insulin lispro.

$\mathrm{O}$ S OBjetivos do TRATAMENTO DO diabetes mellitus (DM) para o paciente com DM tipo l (DMl) visam o desaparecimento dos sintomas, melhorar a qualidade de vida e minimizar o risco de complicações através de um bom controle glicêmico. Na década de 1990, dois fatos importantes implementaram o tratamento do DMl. Primeiro foi a conclusão final de uma série de ensaios clínicos com pacientes com DMl que correlacionavam o controle restrito do DM com menor incidência das complicações crônicas. Um desses estudos, Diabetes Control and Complications Trial (DCCT) (1) mostrou sem questionamento que o controle glicêmico

\section{artigo original}

\author{
Miguel N. Hissa \\ Ana Sofia R. Hissa \\ Veralice M.S. de Bruin
}

Departamento de Medicina Clínica. Faculdade de Medicina, Universidade Federal do Ceará, Fortaleza, CE
Recebido em 11/05/01 Revisado em 13/07/01 Aceito em 18/08/01 
que mais se aproximava do estado não diabético retardava ou desacelerava a progressão das complicações de retina, renal e neurológica. Outro benefício mostrado incluiu a diminuição no risco para a mãe e para o feto durante a gestação (2). Para se conseguir este objetivo glicêmico passou-se a utilizar esquemas terapêuticos que procuravam reproduzir a secreção fisiológica de insulina, utilizando-se múltiplas doses de insulina ou infusão subcutânea contínua de insulina (insulinoterapia intensificada). A insulinoterapia intensificada tinha como objetivo alcançar um estado de euglicemia ou glicemia quase normal através da integração de vários componentes do tratamento do $\mathrm{DM}$ dentro de um estilo de vida individual. Estes componentes deveriam incluir: 1) freqüente monitorização da glicemia capilar; 2) ajuste ativo da dose de insulina, alimentação, $\mathrm{e} /$ ou atividade física, baseado nos resultados da glicemia; 3) utilização dos resultados da glicemia, para individualmente definir os objetivos, 4) Interação continuada entre o paciente com DM e a equipe de saúde.

Todavia, com as insulinas existentes no mercado, os esforços para manter o nível de glicose plasmática tão próximo quanto possível do normal, associavam-se com um aumento do risco de reações hipoglicêmicas, algumas de grande gravidade, e indesejável ganho de peso (1).

O segundo fato importante dessa década, foi a descoberta de moléculas modificadas de insulina, os análogos de insulina e dentre elas, a insulina lispro, que apresentava um perfil de ação mais semelhante à secreção fisiológica das células beta associados a um menor risco de hipoglicemia (3). A insulina é um heterodímero consistindo de duas cadeias, A e B com 21 e 30 aminoácidos respectivamente. As moléculas de insulina humana têm uma tendência natural de autoassociação. Quando duas moléculas ficam muito próximas, forma-se uma ligação reversível entre elas, criando um dímero de insulina. Na presença de zinco, como usado nas formulações de insulina humana (e animal) comercialmente disponíveis, três dímeros se auto-associam para formar um hexâmero (4). Após a injeção subcutânea os hexâmeros de insulina devem se dissociar antes que as moléculas individuais de insulina sejam absorvidas na circulação sistêmica.

O tempo de ação da insulina regular humana depende da velocidade com que os hexâmeros de insulina se dissociam no tecido subcutâneo após sua aplicação. A insulina regular quando administrada por via subcutânea e imediatamente antes de uma refeição, por apresentar uma lenta dissociação, provoca um descompasso entre o seu pico de ação e a velocidade de absorção de carboidrato pela mucosa intestinal.
Como consequiência, poderá desencadear um estado de hiperglicemia pós-prandial seguindo estado de hiperinsulinemia inapropriado e hipoglicemia 4 a 5 horas após a refeição (5). A busca de uma insulina de ação mais rápida levou os pesquisadores a estudar modificações na molécula de insulina, que pudessem apresentar uma tendência reduzida para a auto-associação e, conseqüentemente, uma prontidão aumentada para se dissociar em monômeros.

A insulina lispro é sintetizada pela permutação entre os aminoácidos de números 28 (prolina) e 29 (lisina) da cadeia $\mathrm{B}$ da molécula de insulina. Embora a insulina lispro exista na sua formulação como hexâmero estabilizado com ions de zinco, essa difere da regular pelo fato de seus complexos hexaméricos dissociarem em subunidades monoméricas virtualmente instantaneamente após a injeção no tecido subcutâneo, tornando o seu perfil de absorção mais rápida, início mais rápido e duração de ação mais curta que a insulina humana regular $(3,6,7)$. Apresenta, pois, um início de ação mais rápido (até 15 minutos), um pico máximo em torno de 30 a 90 minutos e um término de ação em 4 horas $(6,7)$. Essa farmacocinética da lispro a credencia como a insulina ideal para ser usada antes das refeições, particularmente quando as mesmas se constituírem de um teor relativamente alto de carboidratos e baixo de lipídeos. Conseqüentemente, a insulina lispro oferece um perfil de tempo de ação mais fisiológico que as insulinas de ação rápida até então disponíveis comercialmente e pode ser considerado um passo na direção da otimização da terapia com insulina.

Atualmente, o tratamento padrão demanda que o médico utilize uma terapia intensificada para o típico paciente com DMl que se encontra em boa saúde. $\mathrm{E}$, numa extensão muito maior do que antes, a insulinoterapia deverá ser individualizada e adaptada para o estilo de vida preferido do paciente. Esse novo tratamento visa instruir o paciente com DM a seguir um padrão alimentar que lhe seja habitual, com ajustes na dose de insulina, ao invés de suscitar a adesão a um programa alimentar e dose de insulina rígidos.

Dois esquemas insulinoterápicos são eficazes em se obter este controle: múltiplas injeções por dia (MID) ou por infusão subcutânea contínua de insulina (ISCI) através de uma bomba infusora.

O protocolo de MID inclui um mínimo de 3 a 4 injeções diárias combinando-se insulinas de ação rápida e intermediária ou prolongada (p.ex., regular e $\mathrm{NPH}$ ). A infusão subcutânea contínua de insulina (ISCI) envolve o uso de pequenos equipamentos mecânicos (bombas), no qual se coloca um reservatório ou uma seringa com insulina de ação rápida ou 
ultra-rápida. Essa seringa é conectada a um cateter de tubo plástico. Ao final do cateter há uma agulha $(27$ gange) ou cânula que é inserida no tecido celular subcutâneo do paciente. O local usual de injeção é o abdome pois a absorção de insulina é mais consistente desse local. A insulina pode ser liberada de duas formas: continuamente, representando a infusão basal e em pulsos (bolus). A infusão basal de insulina geralmente varia de 0,4 a $2 \mathrm{U} /$ hora, podendo se programar até 24 diferentes basais nas $24 \mathrm{hs}$, dependendo do seu usuário. A infusão do bolus é programada e administrada em diferentes horários imediatamente antes das refeições. Na ISCI, a infusão basal procura reproduzir a ação da insulina intermediádia. A dose de insulina administrada em bolus (antes das refeições) é programada individualmente pelos pacientes e seu efeito é análogo à administração por injeção de insulina de ação rápida.

Vários estudos, particularmente em jovens, têm demonstrado que a ISCI e MDI conduzem a resultados semelhantes (8-10). Entretanto, tais estudos foram realizados antes do desenvolvimento de análogos de insulina de ação ultra-rápida. Até o presente momento não há protocolos para recomendação ou seleção do uso de ISCI no lugar de MDI, embora a ISCI seja mais dispendiosa. Algumas vezes, são usados para determinar esta recomendação a preferência e aspectos peculiares do paciente, como motivação, gestação, hipoglicemia assintomática, sensibilidade insulínica extrema, o fenômeno do alvorecer e refeições sem um padrão temporal (11).

Após a introdução no Brasil da tecnologia de bomba de infusão contínua de insulina, pacientes portadores de DMl foram convocados e reunidos em pequenos grupos ou individualmente com a finalidade de explicar os detalhes do tratamento do DM com ISCI. Ênfase foi dado com relação à via de administração e custos. A escolha pela bomba foi decisão dos pacientes e a priori foi explicado que a opção de não usar a bomba, em nada alteraria o seu tratamento.

Nesse trabalho, procuramos estudar o controle glicêmico numa população com DMl, utilizando-se um sistema de infusão subcutâneo contínuo de insulina (bomba de insulina) e um análogo sintético da insulina (insulina lispro).

\section{MATERIAL E MÉTODOS}

Foram relacionados 17 pacientes com DMl que fizeram a opção pela bomba. Todos foram tratados por um período mínimo de 18 meses. Foram excluídos pacientes com insuficiência renal crônica ou retinopa- tia grave (pré-proliferativa ou proliferativa) e crianças abaixo de 10 anos de idade. Não foram observadas desistências do tratamento durante o período em acompanhamento. As características clínicas desses pacientes, antes do início da terapia por bomba, estão discriminadas na tabela 1 .

Todos os pacientes foram tratados ambulatorialmente e avaliados a cada 3 meses por um mesmo profissional. Suporte telefônico foi disponível $24 \mathrm{hs}$ ao dia. Os pacientes nos primeiros 30 dias tiveram um acompanhamento aproximado de 1 a 2 vezes por semana, período destinado a treinamento, ajuste da dose basal e bolus de insulina e orientação relacionado à contagem de carboidratos da alimentação dado por uma nutricionista. Todos os pacientes envolvidos no estudo tiveram a mesma assessoria da nutricionista

Foram utilizados 2 tipos de bombas infusoras: 507C e 508 Minimed $^{\circledR}$ (Sylmar, Califórnia, EUA). A dose diária inicial de insulina correspondeu a $75 \%$ da dose total de insulina prévia (pré-bomba); $50 \%$ dessa quantidade foi administrada na forma de basal e o restante $50 \%$ dividido em 3 bolus iguais administrados antes das refeições. A insulina utilizada na bomba foi a lispro.

Como parâmetros, avaliamos a hemoglobina glicosilada $\left(\mathrm{HbA}_{1} \mathrm{c}\right)$, dosada no início e a cada 3 meses durante 18 meses. O tempo zero foi considerado o momento do envolvimento dos pacientes no estudo. A $\mathrm{HbA}_{1} \mathrm{c}$ foi dosada pelo método HPLC (cromotografia de alta performance - Variant/Bio-RAD). $\mathrm{O}$ valor nor-

Tabela 1. Dados clínicos dos 17 diabéticos tipo 1 antes do início da terapia por ISCl através de bomba.

\begin{tabular}{|c|c|}
\hline Dado clínico & Achado \\
\hline $\operatorname{Sexo}(M: F)$ & $9: 8$ \\
\hline \multicolumn{2}{|l|}{ Idade (anos) } \\
\hline Mediana & 25,0 \\
\hline Media $\pm D P$ & $26,9 \pm 12,4$ \\
\hline Variação & $11-48$ \\
\hline \multicolumn{2}{|c|}{ Duração do diabetes (anos) } \\
\hline Media $\pm D P$ & $14,6 \pm 11,4$ \\
\hline Variação & $1-41$ \\
\hline Peso (kg) & $59,2 \pm 14,2$ \\
\hline $\mathrm{IMC}(\mathrm{kg} / \mathrm{m} 2)$ & $22,5 \pm 2,9$ \\
\hline $\mathrm{HbAlc}(<6.4 \%)$ & $8,3 \pm 1,1$ \\
\hline \multicolumn{2}{|c|}{ Tratamento insulínico prévio } \\
\hline 2 injeções & 6 \\
\hline 3 injeções & 8 \\
\hline 4 injeções & 3 \\
\hline
\end{tabular}


Tabela 2. Resultados (media \pm DP) da terapia com ISCl em 17 pacientes com DM1.

\begin{tabular}{lccc}
\hline Parâmetro & Inicial & 18 meses & $P$ \\
\hline Peso & $59,2 \pm 14,2$ & $59,7 \pm 12,8$ & $\mathrm{NS}$ \\
IMC & $22,5 \pm 2,9$ & $22,28 \pm 2,7$ & $\mathrm{NS}$ \\
Insulina (UI/24h) & $48,8 \pm 23,3$ & $37,6 \pm 17,6$ & $\mathrm{NS}$ \\
Relação insulina/peso (UI/kg) & $0,79 \pm 0,22$ & $0,61 \pm 0,18$ & 0.017 \\
Relação R/NPH versus Bolus/Basal & $0,68 \pm 0,19$ & $1,2 \pm 0,23$ & 0.018 \\
Relação R versus bolus (24h) & $19,8 \pm 0,6$ & $20,5 \pm 0,6$ & $\mathrm{NS}$ \\
Relação NPH versus basal (24h) & $28,9 \pm 0,2$ & $17,0 \pm 0,6$ & 0.008 \\
$\mathrm{HbA}_{1} \mathrm{C}$ & $8,27 \pm 1,06$ & $6,49 \pm 0,53$ & $<0.001$ \\
\hline
\end{tabular}

mal considerado foi de 4,5 a $6,4 \%$. Foram ainda avaliados no início e no final do estudo: 1$)$ o peso $(\mathrm{kg})$ e índice de massa corpórea (IMC, $\mathrm{kg} / \mathrm{m}^{2}$ ): 2) a dose diária total de insulina, calculado pela media diária num período de 3 dias $(\mathrm{U} / 24 \mathrm{~h}) ; 3)$ a dose diária $(\mathrm{U} / 24 \mathrm{~h})$ de insulina regular (R), lispro (Lp) e $\mathrm{NPH}(\mathrm{N}) ; 4)$ a relação entre a insulina $\mathrm{R}$ ou Lp e $\mathrm{N}(\mathrm{R}-\mathrm{Lp} / \mathrm{N}) ; 5)$ a relação entre a dose diária de insulina e peso $(\mathrm{I} / \mathrm{P}$ $\mathrm{U} / \mathrm{kg})$; 6) a dose diária de insulina lispro administrada na forma de infusão contínua basal (basal $-\mathrm{U} / 24 \mathrm{~h})$ e antes das refeições (bolus - U/24h) nos pacientes tratados com ISCI; 7) a relação entre a dose diária de insulina administrada na forma de bolus e basal (B/B).

Os dados foram analisados usando um software para Windows (SPSS versão 7.5, Chicago). Foi aplicado o teste de Kolmogorov-Smirnov para testar a normalidade das amostras. Os resultados paramétricos foram expressos através de média e desvio padrão (DP). Para comparação das variáveis entre os grupos foi utilizado ANOVA seguido do teste de comparação múltipla de Dunnett quando aplicável. O intervalo de confiança foi significante se $\mathrm{p}<0,05$.

\section{RESULTADOS}

O estudo compreendeu a análise de 17 pacientes com $\mathrm{DMl}$, submetidos a terapia por ISCI por um período de 18 meses. Os dados relativos à $\mathrm{HbA}_{1 \mathrm{c}}$, peso, IMC e dosagem de insulina estão discriminados na tabela 2 .

\section{Hemoglobina glicosilada}

A $\mathrm{HbA}_{1} \mathrm{c}$ foi dosada no início do período, época em que os pacientes passaram a fazer parte da população estudada, e a cada 3 meses durante 18 meses consecutivos (tabela 3). Após o início da ISCI, a $\mathrm{HbA}_{1}$ c média diminuiu significativamente já no primeiro trimestre, de $8,27 \%$ para $7,26 \%(\mathrm{p}<0,001)$ e durante todo o período de observação manteve-se significativamente mais baixa do que o valor inicial (tabela 4). Ao final de 18 meses encontrava-se em 6,49\% (figura 1).

Tabela 3. Niveis de $\mathrm{HbA}_{1 \mathrm{c}}$ nos 17 pacientes com DM1 tratados com ISCI no período de 18 meses.

\begin{tabular}{lcccc}
\hline $\begin{array}{l}\text { Período } \\
\text { (mês) }\end{array}$ & \multicolumn{4}{c}{$\mathrm{HbA}_{1} \mathrm{C}$} \\
\cline { 2 - 5 } & Mínimo & Máximo & Média & $\mathrm{DP}$ \\
\hline 0 & 6,43 & 10,70 & 8,27 & 1,06 \\
3 & 5,80 & 8,60 & 7,26 & 0,80 \\
6 & 5,90 & 8,80 & 6,87 & 0,77 \\
9 & 5,77 & 8,50 & 6,76 & 0,61 \\
12 & 5,25 & 8,10 & 7,07 & 0,71 \\
15 & 5,80 & 7,45 & 6,63 & 0,60 \\
18 & 5,50 & 7,50 & 6,49 & 0,53 \\
\hline
\end{tabular}

Tabela 4. Valores médios da $\mathrm{HbA}_{1 \mathrm{c}}$ nos 17 pacientes com DMl durante o período estudado (18 meses).

\begin{tabular}{lccccc}
\hline Período(I) & Período $(J)$ & Média (\%) & Diferença Média (I-J) & Erro padrão & P-valor* \\
\hline & Inicial & 8,27 & & & \\
3 meses & Inicial & 7,26 & $-1,01$ & 0,259 & $<0,001$ \\
6 meses & Inicial & 6,87 & $-1,39$ & 0,733 & $<0,001$ \\
9 meses & Inicial & 6,76 & $-1,51$ & 0,688 & $<0,001$ \\
12 meses & Inicial & 7,07 & $-1,20$ & 0,268 & $<0,001$ \\
15 meses & Inicial & 6,63 & $-1,64$ & 0,279 & $<0,001$ \\
18 meses & Inicial & 6,49 & $-1,77$ & 0,259 & $<0,001$ \\
\hline
\end{tabular}

*Teste de Dunnet $(p<0,05)$ 


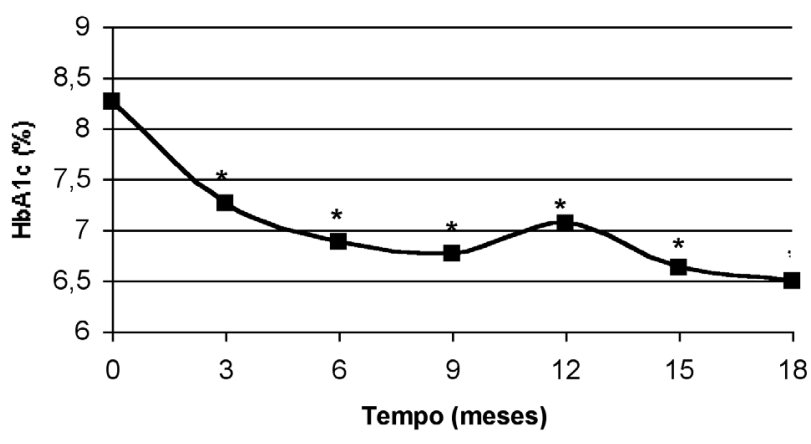

Figura 1. Evolução da hemoglobina glicosilada $\left(\mathrm{HbA}_{1 \mathrm{c}}\right)$ média dos 17 pacientes com DMl, dosada a cada 3 meses durante 18 meses. ${ }^{*}(p<0,05)$.

\section{Peso e IMC}

O peso médio e o IMC não variaram significativamente entre o período inicial e final nos 3 grupos estudados.

\section{Dose de insulina}

Os dados referentes a utilização de insulina estão relacionados na tabela 2 . Não houve variação significativa entre a dose média de insulina inicial e após 18 meses em tratamento com ISCI. Entretanto, a relação insulina/peso apresentou uma variação significativa com a ISCI $(p=0,017)$.

\section{Relação insulina R-Lp/N e Bolus/Basal}

A dose média diária de insulina rápida ( $\mathrm{R}$ ou $\mathrm{Lp}$ ) (19,8U) inicial (pré-bomba) foi semelhante a dose média diária da insulina administrada sob a forma de bolus $(20,5 \mathrm{U})(\mathrm{p}=0,619)$. Todavia, a quantidade média diária da insulina administrada na forma de basal (17U/24hs) foi significativamente menor que a dose média diária de insulina intermediária $(\mathrm{N})$ administrada antes do tratamento por ISCI (28,9U/24hs) $(\mathrm{p}=0,008)$. Comparando a relação entre as quantidades diária de insulina de ação rápida e intermediária ( $\mathrm{R}-\mathrm{Lp} / \mathrm{N})$ no início, com a relação entre a quantidade diária de insulina administrada nas formas de bolus e basal (B/B) no final do período, observamos um aumento significativa após 18 meses $(\mathrm{p}=0,018)$.

\section{Curso clínico}

Nenhum episódio severo de hipoglicemia que necessitasse ajuda de terceiros, ocorrência de cetoacidose ou infecção local que demandasse tratamento com antibiótico foram observados. Não houve necessidade de hospitalização para o iniciar o tratamento, bem como no curso desse.

\section{DISCUSSÃO}

Durante os últimos anos a intenção de se obter um nível glicêmico quase normal como meta terapêutica do DMl tem sido estimulada. A insulinoterapia intensificada, quer através de sua infusão subcutânea contínua quer de múltiplas aplicações diárias, passou a representar importante componente na obtenção deste controle (1). Avaliamos, nesse trabalho, os resultados terapêuticos de 17 pacientes com DMl tratados durante 18 meses com bomba de infusão subcutânea contínua de insulina.

A análise dos resultados demonstra que com o uso de ISCI houve uma melhora no controle glicêmico, avaliado pelos resultados da $\mathrm{HbA}_{\mathrm{l}} \mathrm{c}$ (figura 1). Observamos que, desde o primeiro trimestre após o início da ISCI, houve uma redução média no valor absoluto da hemoglobina glicosilada estatisticamente significativo $(\mathrm{p}<0,001)$. Poder-se-1́a atribuir essa redução à empolgação do usuário da bomba, pelo uso de uma nova modalidade de insulinoterapia. Contudo, quando comparamos as demais dosagens de $\mathrm{HbA}_{1} \mathrm{c}$ com o valor inicial, observamos que ela se manteve significativamente abaixo durante todo o período de 18 meses. Ao final, dos 17 pacientes submetidos a tratamento com bomba de infusão subcutânea contínua de insulina, 13 (76,4\%) apresentaram níveis de $\mathrm{HbA}_{1} \mathrm{c}$ menor que $7 \%$ e 4 $(23,6 \%)$ entre $7 \%$ e $7,5 \%$. Dezesseis pacientes apresentaram um decréscimo na $\mathrm{HbA}_{1} \mathrm{c}$ superior a $10 \%$ (entre 10 e $20 \%$ em 7 , entre 20 e $30 \%$ em 6 e acima de $30 \%$ em 3 ); somente 1 teve uma redução de $7,5 \%$ no seu valor de $\mathrm{HbA}_{1} \mathrm{c}$. Entretanto esse paciente apresentava valores inicial e final de $\mathrm{HbA}_{1} \mathrm{c}$ respectivamente de 6,43\% e 5,95\%. A redução no valor absoluto médio da $\mathrm{HbA}_{1} \mathrm{c}$ foi de $1,78 \%$. Os nossos resultados comparam-se a de outros previamente publicados e que demonstraram uma redução da hemoglobina glicosilada de 1 a 3,4\% (9,1214). Se considerarmos os dados do DCCT sob o declínio do valor absoluto da hemoglobina glicosilada de 1\%, poderemos estimar que mantendo-se essa meta, os nossos pacientes se beneficiarão com uma redução de pelo menos $43 \%$ no risco de retinopatia, $48 \%$ de nefropatia e $38 \%$ de neuropatia (16). E, novamente levando-se em conta os resultados apresentados pelo DCCT, de que uma redução de $10 \%$ no valor da hemoglobina glicosilada (ex: de 9\% para 8,1\%) estará associado com uma redução de $44 \%$ na progressão de retinopatia, poderíamos então projetar o grande benefício trazido pela ISCI aos nossos pacientes.

Nesse estudo, após 18 meses, o tratamento com ISCI levou a uma redução da dose media diária de insulina da ordem de $23 \%$, entretanto, este valor não foi significativo $(p=0,124)$. Esse dado está de acordo 
com outros resultados publicados previamente (1619), embora alguns tenham mostrado uma redução significativa na dose media diária de insulina $(16,20)$. No nosso estudo, as maiores alterações com relação a dose diária de insulina diz respeito à: primeiro, a diminuição da insulina administrada não relacionada à alimentação (insulina basal). Quando comparamos a dose diária de NPH $(28,9 \mathrm{U})$ no início do estudo com a dose diária da insulina basal $(17 \mathrm{U})$ ao final, observamos um decréscimo significativo $(\mathrm{p}=0,008)$; e segundo, ao aumento relativo da insulina administrada com a alimentação (bolus). Nós expressamos esta observação como a razão bolus/basal $(1,2)$ que foi superior a razão entre as insulinas de ação rápida/intermediária [R-Lp/NPH] $(0,68)$ antes do início do estudo. Esta diferença foi estatisticamente significante $(\mathrm{p}=0,018)$. Conforme dados publicados previamente, esquemas terapêuticos que aumentem esta razão acima de 1,0 têm demonstrados serem mais eficazes (20).

Nestes pacientes observamos um decréscimo da relação insulina/peso após 18 meses. Esse fato que poderia ser atribuído tanto à redução da dose de insulina como ao aumento de peso. Surpreendentemente, nenhuma alteração significativa no peso foi observada; a relação insulina/peso variou de $0,79 \mathrm{U} / \mathrm{kg}$ (início) para $0,61 \mathrm{U} / \mathrm{kg}\left(18^{\circ}\right.$ mês $)$, tendo sido esta diferença significativa $(p=0,017)$. Se levarmos em conta que o peso manteve-se estável, podemos concluir que a insulina foi usada mais efetivamente. Esse dado quando analisado conjuntamente com o observado em relação ao aumento da razão bolus/basal sugere que a administração de um percentual de insulina na forma de bolus pré-prandial poderá levar a um melhor controle glicêmico sem ganho de peso. Estudos prévios relatam um aumento de peso de 1,7 a $3,5 \mathrm{~kg}$ em 1 ano $(1,21)$.

A redução global dos valores glicêmicos pela insulina conduz a um risco aumentado de hipoglicemia. No DCCT (1), a melhoria do controle glicêmico através de terapia intensificada foi acompanhada de uma incidência de hipoglicemia severa 3 vezes superior quando comparado ao grupo de pacientes em tratamento convencional. No nosso estudo, não tivemos quaisquer episódios de hipoglicemia severa com ISCI, apesar dos pacientes manterem um controle mais intenso da sua glicemia, avaliado pela dosagem de hemoglobina glicosilada. Os nossos dados são comparáveis aos de várias análises publicadas previamente que evidenciaram uma redução na freqüência de coma hipoglicêmico com ISCI $(22,23)$, embora esta mesma observação não seja compartilhada por outros $(24,25)$.

Uma das desvantagens da ISCI é a susceptibilidade desses pacientes desenvolverem rapidamente um estado ceto-acidótico (26). Um estudo de metaanálise identificou que a ceto-acidose diabética era mais comum em pacientes com tratamento de ISCI do que naqueles em tratamento convencional (27). Pelas características fármaco-dinâmicas da insulina lispro comparada com a insulina regular, tendo ela uma duração de ação mais curta, a tendência a precipitar um estado de ceto-acidose após a interrupção acidental de sua infusão é maior $(28,29)$. No nosso estudo não observamos no período de 18 meses qualquer estado de ceto-acidose.

A base dos nossos achados para melhoria do controle glicêmico é provavelmente multifatorial: A liberação de insulina por intermédio de uma bomba oferece a maneira mais fisiológica de tratamento insulínico correntemente avaliável, pois procura reproduzir através de uma infusão contínua, a insulinemia basal, e através de bolus administrado antes das refeições, os picos fisiológicos de insulina que acontecem nessa hora. A ISCI permite não somente alterações flexíveis na insulina basal para ajudar a prevenção de hipoglicemia como também um aumento de sua infusão para suprir o requerimento aumentado durante o fenômeno do alvorecer (30).

\section{CONCLUSÕES}

Concluímos pelos dados obtidos em nosso estudo, que a ISCI no tratamento do DMl é eficaz, reduzindo significativamente os níveis de hemoglobina glicosilada e, portanto, melhorando o controle glicêmico. Permite uma utilização mais efetiva da insulina, com um incremento da insulina administrada durante as refeições e uma redução da insulina basal (não relacionada à alimentação). Não afeta adversamente o peso, requerendo menos unidades de insulina por $\mathrm{kg}$ de peso. É um procedimento seguro podendo ser aplicável ambulatorialmente. Não aumenta a incidência de hipoglicemia severa nem de cetoacidose.

\section{AGRADECIMENTOS}

À Norma de Carvalho Linhares, chefe da Biblioteca da Faculdade de Medicina da UFC e aos nossos pacientes com DM por sua participação nesse projeto.

\section{REFERÊNCIAS}

1. The Diabetes Control and Complications Trial. Research Group. The effect of intensive treatment of diabetes on the development and progression of long-term complications in insulin-dependent diabetes mellitus. N Engl J Med 1999;329:977-86. 
2. The Diabetes Control and Complications Trial. Research Group. Pregnancy outcomes in the diabetes control complications trial. Am J Obstet Gynecol 1996; 174:1343-53.

3. Torlone E, Fanelli C, Rambotti AM, Kassi G, Modarelli F, di Vincenzo A, et al. Pharmacokinetics, pharmacodynamics, and glucose counterregulation following subcutaneous injection of monomeric insulin analogue (Lys(B28), Prol(B29)) in DMID. Diabetologia 1994;37:713-20.

4. Ciszak E, Beals JM, Baker JC, Frank BH, Carter ND, Smith GD. Role of C-terminal B-chain residues in insulin assembly: Structure of hexameric Lys(B28), Pro(B29)-human insulin. Structure 1995;3:615-22.

5. Dimitriadis GD, Gerich JE. Importance of timing of preprandial subcutaneous insulin administration in the management of diabetes mellitus. Diabetes Care 1983;6:374-77.

6. Radziuk JM, Davis JC, Pye WS, Schields JE, Dimarchi RD, Chance RE. Bioavailability and bioeffectiveness of subcutaneous human insulin and two of its analogs LysB28,ProB29-human insulin and AspB10, LysB28,ProB29human insulin - assessed in a conscious pig model. Diabetes 1997; $46: 548-56$.

7. Howey DC, Bosher RR, Brunelle RL, Woodworth JR. (Lys(B28),Pro(B29))-Human insulin: A rapidly absorbed analogue of human insulin. Diabetes 1994;43:396-402.

8. Mecklenburg RS, Benson EA, Benson JW Jr, Blumenstein BA, Fredlund PN, Guinn TS, et al. Long-term metabolic control with insulin pump therapy: report of experience with 127 patients. N Engl J Med 1985:313:465-68.

9. Bode BW, Steed RD, Davidson PC. Reduction in severe hypoglycemia with long-term continuous subcutaneous insulin infusion in type 1 diabetes. Diabetes Care 1996; 19:324-27.

10. DCCT Reseach Group. Epidemiology of severe hypoglycemia in the diabetes control and complication trial. The DCCT Research Group. Am J Med 1991;90:450-59.

11. Farkas-Hirsch R, Hirsch IB. Continuous subcutaneous insulin infusion: a review of the past and its implementation for the future. Diabetes Spect 1994;7:80-84.

12. Ronn B, Mathiesen ER, Vang L, Lorup B, Deckert T. Evaluation of insulin pump treatment under routine conditions. Diabetes Res Clin Pract 1987:3:191-96.

13. Chantelau E, Spraul M, Muhlhauser I. Long-term safety, efficacy and side-effects of continuous subcutaneous onsulin infusion treatment for type 1 (insulin-dependent) diabetes mellitus: a one centre experience. Diabetologia 1989:32:421-26.

14. Koznarová R, Pelikánova T, Dryáková M. Results of longterm follow-up of patients treated by continuous subcutaneous insulin infusion. (abstract). Diabetes Res Clin Pract 2000;50 (suppl. 1):S79

15. Diabetes Control and Complication Trial. Study Group. The absence of a glycemic threshold for the development of long-term complications: the perspective of Diabetes Control and Complications Trial (DCCT). Diabetes 1996;45:1289-98.

16. Schiffrin A, Belmonte MM. Comparison between continuous subcutaneous insulin infusion and multiple injection of insulin: a one year prospective study. Diabetes 1982;31:255-64.

17. Wredling $R$, Lins $P-E$, Adamson U. Factors influencing clinical outcome of continuous subcutaneous insulin infusion in routine practice. Diabetes Res Clin Pract 1993: 19:659-67.
18. Bode B, Steed D, Davidson P. Long-term pump use and SMBG in 205 patients (abstract). Diabetes 1994;43 (suppl. 1):691.

19. Wredling R, Hannerz L, Johansson U-B. Variability of blood glucose levels in patients treated with continuous subcutaneous insulin infusion: a pilot study. Pratical Diabetes Int 1997; 14:5-8

20. Crawford LM, Sinha RN, Odell RM, Comi RJ. Efficacy of insulin pump therapy: mealtime delivery is the key factor. Endocr Pract 2000;6:239-43.

21. Home PD, Capaldo B, Burrin JM, Worth R, Alberti KGA. Cross-over comparison of continuous subcutaneous insulin infusion (CSII) against multiple insulin injections in insulin-dependent diabetics subjects: improved control with CSII. Diabetes Care 1982;5:466-71.

22. Dahl-Jørgensen $\mathrm{K}$, Brinchmann-Hansen $\mathrm{O}$, Hanssen $\mathrm{KF}$, Ganes T, Kierulf P, Smeland E, et al. Effect of near normoglycaemia for two years on progression of early diabetic retinopathy, nefropathy, and neuropathy: the Oslo study. Br Med J 1986;293:1 195-99.

23. Eichner HL, Selam JL, Holleman CB, Worcester BR, Turner DS, Charles MA. Reduction of severe hypoglycaemic events in type I (insulin-dependent) diabetic patients using continuous subcutaneous insulin infusion. Diabetes Res 1988;8:189-93.

24. Mecklenburg RS, Benson EA, Benson JW Jr, Fredlund PN, Guinn T, Metz RJ, et al. Acute complications associated with insulin infusion pump therapy: report of experience with 161 patients. JAMA 1984;252:3265-69.

25. Bending JJ, Pickup JC, Keen H. Frequency of diabetic ketoacidosis and hypoglycemic coma during treatment with continuous subcutaneous insulin infusion: audit of medical care. Am J Med 1985;79:685-91.

26. American Diabetes Association. Position Statement: Continuous subcutaneous insulin infusion. Diabetes Care 1991;14(Suppl.1):S34-S35.

27. Wang PH, Lau J, Chalmers TC. Meta-analysis of effect of intensive blood glucose control on late complications of type 1 diabetes. Lancet 1993;341:1306-09.

28. Pein M, Hinselmann C, Pfützner A, Dreyer M. Catheter disconnection in type 1 diabetic patients treated with CSII: comparison of insulin lispro and human regular insulin. Diabetologia 1996;39(suppl. 1):847.

29. Reichel A, Rietzsch H, Köhler HJ, Pfutzner A, Gudat U, Schulze J. Cessation of insulin infusion at night-time during CSIl-therapy: comparison of regular human insulin and insulin lispro. Exp Clin Endocrinol Diabetes 1998; 106:168-72.

30. Haakens K, Hanssen KF, Dahl-Jørgensen K, Vaaler S, Aagenaes $O$, Mosand $R$. Continuous subcutaneous insulin infusion (CSII), multiple injections (MI) and conventional insulin therapy (CT) in self-selecting insulindependent diabetic patients: a comparison of metabolic control, acute complications and patient preferences. J Intern Med 1990;228:457-64.

\section{Endereço para correspondência:}

Miguel Nasser Hissa

R. Ana República do Líbano, 647

60160-140 Fortaleza, Ceará

Fax: (085) 242-3241

e-mail: miguelnh@secrel.com.br 